\title{
VIPER: A Genetically-Encoded Tag for Fluorescence and Electron Microscopy
}

Julia K. Doh ${ }^{1 *}$, Jonathan D. White ${ }^{1}$, Hannah K. Zane ${ }^{1}$, Young Hwan Chang ${ }^{1}$, Claudia S. López ${ }^{1}$, Caroline A. Enns, ${ }^{1}$ and Kimberly E. Beatty ${ }^{1}$

${ }^{1}$ Oregon Health \& Science University, Portland, Oregon, USA

*Corresponding author: dohj@ohsu.edu

Studying biology at high magnification is primarily enabled by fluorescence and electron microscopy (EM). These two methods rely on distinct mechanisms of contrast: light and electron density. Correlative light and EM (CLEM) benefits from the target specificity of fluorescence with the high resolution and ultrastructural data that comes from EM. In order to see a protein by these methods, you must tag the protein with a reporter molecule. There are many available genetically-encoded fluorescent tags, such as fluorescent proteins [1], or SNAP tag and HaloTag [2, 3]. By contrast, there are far fewer genetically-encoded protein tags for EM [4, 5], and even fewer that are compatible with both fluorescence microscopy and EM.

In recognizing this new imaging opportunity, we developed a genetically-encoded tag for high resolution imaging by fluorescence and EM, named Versatile Interacting Peptide (VIP) Tags. VIP tags are coiled-coil peptides, where one coil peptide is expressed as a genetically-encoded fusion to the protein of interest (Figure 1). The partner coil labels the tagged protein with a reporter through heterodimer formation. The VIP probe peptides were designed to have a single cysteine residue that acts as a bioconjugation site for attachment to fluorophores or EM labels. Our first VIP tag, VIP Y/Z (comprised of CoilY and CoilZ), enabled selective fluorophore labeling of proteins on living cells [6].

In our latest publication we described VIPER [7], which is comprised of a CoilE tag and CoilR probe peptide. First, we verified the specificity of VIPER by labeling various subcellular targets in fixed cells. We used VIPER to label mEmerald-fused actin, mitochondria ("Mito"), or histone H2B in fixed, permeabilized cells. The fluorescent protein mEmerald confirmed canonical morphology for each organelle structure and was colocalized with VIPER signal for CoilE-tagged targets. VIPER labeling was specific and we did not observe non-specific or off-target labeling with the CoilR probe peptide.

Next we used VIPER to image transferrin receptor 1 (TfR1), a transmembrane protein that undergoes clathrin-mediated endocytosis. We detected the receptor, TfR1-CoilE, with CoilR-Cy5 and the ligand, transferrin, fluorescently labeled with AF488 (Tf-AF488). In live cells, VIPER-labeled TfR1 had normal Tf-binding and internalization rates, indicating the biocompatibility and efficacy of VIPER. VIPER allowed for tracking of multiple populations of receptors at a time, such as in pulse-chase labeling.

We developed VIP tags to enable specific protein imaging by multi-scale microscopy, namely CLEM. VIPER facilitated the dual imaging of TfR 1 by fluorescence and EM through the use of Qdots, which are fluorescent and electron dense. We labeled TfR1-CoilE with CoilR-biotin, which allowed us to detect the receptor through streptavidin-Qdot655. We imaged Tf and TfR1 by fluorescence before imaging at 65,000X magnification by scanning EM (Figure 2). We algorithmically quantified the labeling and found $110 \pm 34$ Qdots $/ \mu \mathrm{m}^{2}$ on average for TfR1-CoilE and 0 Qdots $/ \mu \mathrm{m}^{2}$ for untagged TfR1, highlighting the specificity of the technology. We additionally compared VIPER to immunolabeling, the primary labeling method for CLEM, and VIPER outperformed four out of five antibodies tested. 
VIP tags, including VIPER, are a new technology for imaging proteins at high resolution and across different platforms. We demonstrated the application range of VIPER by imaging various cellular proteins in both live and fixed cells. Most notably, VIPER is a new protein tag for CLEM applications.

\section{References:}

[1] BNG Giepmans et al., Science 312 (2006), p. 217.

[2] A Gautier et al., Chem. Biol. 15 (2008), p. 128.

[3] GV Los et al., ACS Chem. Biol. 3 (2008), p. 373.

[4] JD Martell et al., Nat. Biotechnol. 30 (2012), p. 1143.

[5] X Shu et al., PLoS Biol. 9 (2011), p. e1001041.

[6] HK Zane et al., ChemBioChem (2017) p. 470.

[7] JK Doh et al., Proc. Natl. Acad. Sci. (2018), p. 12961.

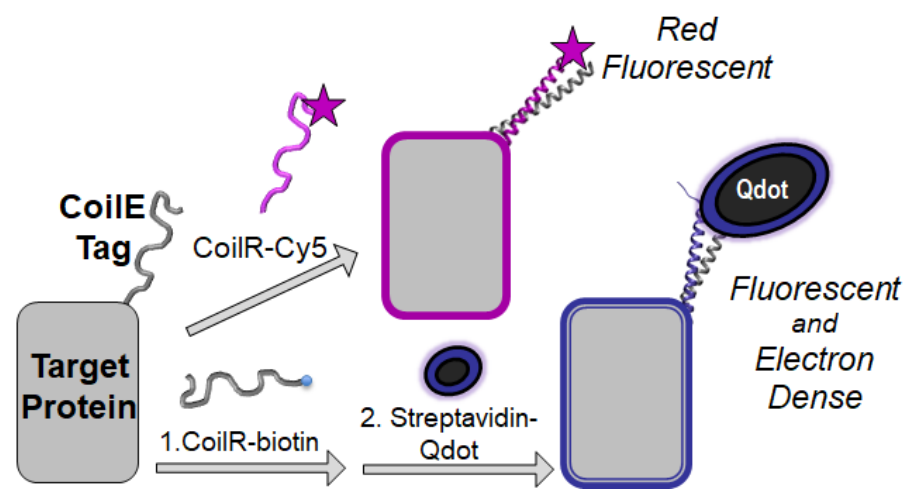

Figure 1. VIPER labeling comprises of a genetically-encoded CoilE tag, which is detected upon dimerization with a CoilR probe peptide. Reporter molecules can be fluorescent or electron-dense. Figure adapted from J. K. Doh et al., Proc. Natl. Acad. Sci. (2018), p. 12961-12966.
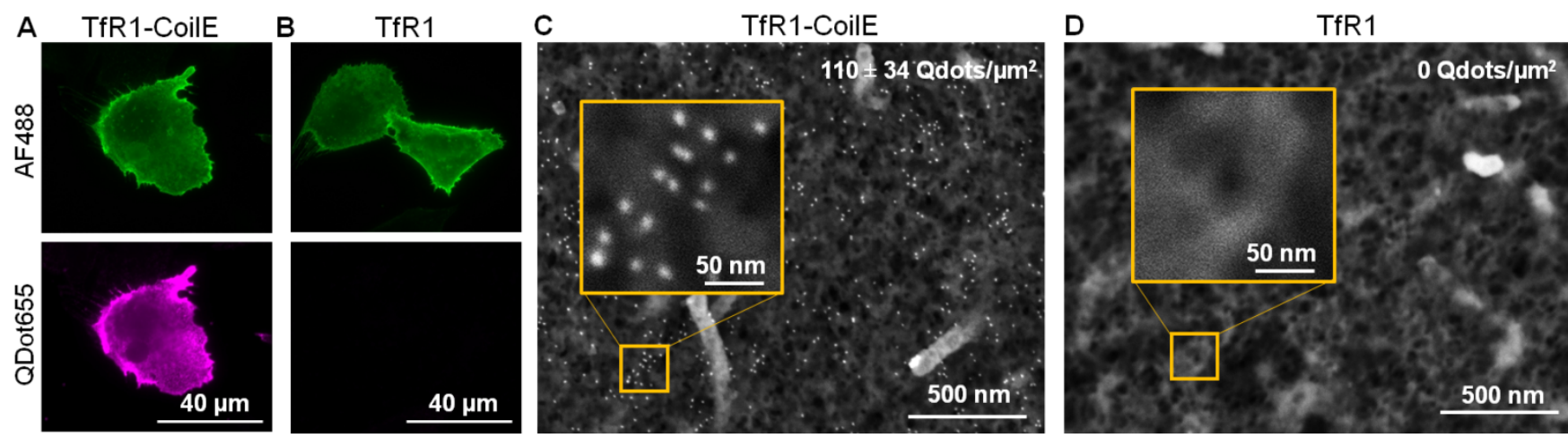

Figure 2. CHO TRVb cells expressing TfR1 or TfR1-CoilE were treated with Tf-AF488 and CoilRbiotin, which was detected by streptavidin-Qdot655. Imaging by fluorescence showed that cells bound Tf-AF488 (2A-2B), but only TfR1-CoilE was labeled by Qdot655 (2A). When imaged at 65,000X magnification (2C-2D), cells expressing TfR1-CoilE labeled by VIPER showed Qdots dispersed across the cell, while cells expressing TfR1 without CoilE had virtually no Qdots. Qdots appear as white dots over the dark gray cell surface. Cell protrusions appear in light gray. Figure adapted from $J$. K. Doh et al., Proc. Natl. Acad. Sci. (2018), p. 12961-12966. 